\title{
ON THE CONSTRUCTION OF CONVERGENT ITERATIVE SEQUENCES OF POLYNOMIALS
}

\author{
QIU WEIYUAN
}

(Received 24 December 1987; revised 11 July 1988)

Communicated by R. O. Vyborny

\begin{abstract}
We answer two conjectures suggested by Zalman Rubinstein. We prove his Conjecture 1, that is, we construct convergent iterative sequences for $f_{m}^{-1}(z)$ with an arbitrary initial point, where $f_{m}(z)=z+z^{m}$ with $m \geq 2$. We also show by several counterexamples that Rubinstein's Conjecture 2 is generally false.
\end{abstract}

1980 Mathematics subject classification (Amer. Math. Soc.) (1985 Revision): 30 D 05.

\section{Introduction}

Zalman Rubinstein constructed convergent iterative sequences for the polynomials $f(z)=z+z^{m}, m \geq 2$, with initial point in the lemniscate $\left\{z|| f^{\prime}(z) \mid\right.$ $\leq 1\}$ by variational methods. His main results showed that for every point $z_{0} \in\left\{z|| f^{\prime}(z) \mid \leq 1\right\}$, the iterative sequence $z_{n+1}=f\left(z_{n}\right), n=0,1, \ldots$, converges to 0 as $n \rightarrow \infty$. In the particular case $m=2$, convergent iterative sequences were constructed also for $f^{-1}(z)$ with an arbitrary initial point. For the case $m>2$, and more generally, for polynomials with positive real coefficients, the following two conjectures were mentioned in [1].

CONJECTURE 1. Let $f(z)=z+z^{m}, m \geq 2$. There exists a determination of $f^{-1}(z)$ such that for every $z_{0} \in \mathbb{C}$ the sequence $z_{n}=f^{-1}\left(z_{n-1}\right)$ tends to zero as $n \rightarrow \infty$.

This work was supported by the Science Fund of the Chinese Academy of Science.

(C) 1989 Australian Mathematical Society 0263-6115/89 \$A2.00+0.00 
CONJECTURe 2. Let $f(z)=z+a_{2} z^{2}+\cdots+a_{m} z^{m}$ be of degree $m \geq 2$, and assume that $a_{k} \geq 0$ for all $k$. Then for every $z_{0}$ such that $\left|f^{\prime}\left(z_{0}\right)\right| \leq 1$, the sequence $z_{n+1}=f\left(z_{n}\right)$ converges.

In this paper, we will discuss the above two problems. We will show that Conjecture 1 is true, while Conjecture 2 is generally false, by way of several counterexamples.

\section{Definitions and lemmas}

We need some results of the Fatou and Julia theory of iteration ([3], [4] and [5]; also see [2]). Let $f(z)$ be a polynomial. Denote $f^{n}=f \circ f \circ \cdots \circ f$ as the $n$th order iteration of $f$. The Fatou set $F$ of $f$ is the maximal open set in which $\left\{f^{n}\right\}$ is a normal family. The Julia set $J$ of $f$ is the complement of $F$. The point $z$ is called an $n$th order periodic point if $f^{n}(z)=z$ and $f^{k}(z) \neq z$ for all $0<k<n$. Such an $n$th order periodic point $z$ is called attractive (repulsive or rationally indifferent respectively) if $\left|\left(f^{n}\right)^{\prime}(z)\right|<1\left(\left|\left(f^{n}\right)^{\prime}(z)\right|>1\right.$ or $\left(f^{n}\right)^{\prime}(z)$ is a root of unity respectively). We also call $\left\{f^{n}(z)\right\}$ a forward orbit of $f$ at $z$, and denote by $f^{-n}(z)$ the inverse images of $f^{n}$ at $z$, for $n=1,2, \ldots$ Every branch of $f^{-n}(z)$ on a domain is denoted by $f_{j}^{-n}(z)$.

The following results of Fatou and Julia will be used.

(1) $F$ is open. $J$ is perfect and non-empty. $F$ and $J$ are completely invariant under $f$, that is, $f(F)=f^{-1}(F)=F$, etc.

(2) The Julia set coincides with the closure of the set of repulsive periodic points.

(3) Every attractive periodic point is in $F$ and every repulsive or rational indifferent periodic point in $J$.

(4) If $f$ is a polynomial, then the unbounded component $A(\infty)$ of $F$ is exactly the set of all points whose iterative sequences tend to infinity.

(5) If $z_{0}$ is not a limit point of the forward orbit of some point $z \notin J$, then every accumulation point of $\left\{f^{-n}(z)\right\}$ belongs to $J$.

(6) Let $\left\{f_{j}^{-n}(z)\right\}_{j, n}$ be any infinite set of inverse branches which are holomorphic in a domain $D$, and suppose that there exists an open subset of $D$ containing no limit points of the forward orbit of any point $z \notin J$. Then $\left\{f_{j}^{-n}(z)\right\}$ is normal in $D$ and every convergent subsequence tends to a constant. 
Now suppose that $g(z)=z+a_{m} z^{m}+\cdots$ is a power series analytic at the origin. For $0<\theta<\pi / 2$ and sufficiently small $\rho>0$, we define the domain

$$
\begin{aligned}
D(j, \theta, \rho)=\left\{z|0<| z \mid<\rho,-\gamma-\frac{(2 j-2) \pi}{m-1}-\frac{\pi-\theta}{m-1}\right. & \\
<\arg z & \left.<-\gamma-\frac{(2 j-2) \pi}{m-1}+\frac{\pi-\theta}{m-1}\right\}
\end{aligned}
$$

for $j=1,2, \ldots, m-1$ and the "star domain" $D(\theta, \rho)=\bigcup_{j=1}^{m-1} D(j, \theta, \rho)$, where $\gamma$ is a constant satisfying $-a_{m} \exp \{-i \gamma(m-1)\}>0$.

LEMMA 1 [6, Lemma 9]. Let $g(z)=z+a_{m} z^{m}+\cdots$ be analytic at the origin. Then for given $0<\theta<\pi / 2$ and sufficiently small $\rho>0$, we have $g(D(\theta, \rho)) \subset$ $D(\theta, \rho)$ and the iteration $g^{n}(z)$ converges to zero locally uniformly in $D(\theta, \rho)$.

Lemma 2. Let $f(z)=z+z^{m}$. Then $\left\{z \mid z^{m-1} \in \mathbf{R}\right\}$, which we abbreviate to $\left\{z^{m-1} \in \mathbf{R}\right\}$, and $\left\{z^{m-1}>0\right\}$ are both invariant under $f$, and $\left\{z^{m-1}>0\right\} \subset$ $A(\infty)$.

Proof. If $z=\rho e^{k \pi i /(m-1)} \in\left\{z^{m-1} \in \mathbf{R}\right\}, 0 \leq \rho<+\infty$, then

$$
f(z)=\rho e^{k \pi i /(m-1)}\left(1 \pm \rho^{m-1}\right) \in\left\{z^{m-1} \in \mathbf{R}\right\} .
$$

If $z=\rho e^{2 k \pi i /(m-1)} \in\left\{z^{m-1}>0\right\}, 0<\rho<+\infty$, then

$$
(f(z))^{m-1}=\left(\left(\rho+\rho^{m}\right) e^{2 k \pi i /(m-1)}\right)^{m-1}=\left(\rho+\rho^{m}\right)^{m-1}>0 .
$$

These show that $\left\{z^{m-1} \in \mathbf{R}\right\}$ and $\left\{z^{m-1}>0\right\}$ are both invariant under $f$.

Because $f(z)=\left(|z|+|z|^{m}\right) e^{2 k \pi i /(m-1)}$ for $z \in\left\{z^{m-1}>0\right\}$, and also $|f(z)|=$ $|z|+|z|^{m} \geq|z|$, we have by induction that

$$
\begin{aligned}
f^{n}(z) & =\left|f^{n-1}(z)\right|\left(1+\left|f^{n-1}(z)\right|^{m-1}\right) e^{2 k \pi i /(m-1)} \\
& =f^{n-1}(z)\left(1+\left|f^{n-1}(z)\right|^{m-1}\right) \\
& =z \prod_{k=0}^{n-1}\left(1+\left|f^{k}(z)\right|^{m-1}\right) .
\end{aligned}
$$

Hence

$$
\left|f^{n}(z)\right| \geq|z|\left(1+|z|^{m-1}\right)^{n}=\rho\left(1+\rho^{m-1}\right)^{n},
$$

which tends to infinity as $n \rightarrow \infty$, that is $\left\{z^{m-1}>0\right\} \subset A(\infty)$, from Result 4 above.

LEMMA 3. Let $l_{k}=\left\{z \mid z=\rho e^{(2 k+1) \pi i /(m-1)},-\infty<\rho<+\infty\right\}, k=1,2, \ldots$, $m-1$, be a straight line in $\left\{z^{m-1} \in \mathbf{R}\right\}$, and let $h_{k}$ be the subset of $l_{k}$,

$$
h_{k}=\left\{z \mid z=\rho e^{(2 k+1) \pi i /(m-1)}, \rho>\rho_{0}\right\},
$$


where $\rho_{0}=((m-1) / m)(1 / m)^{1 /(m-1)}$. Then if $m$ is even, all $m$ branches of $f^{-1}\left(h_{k}\right)$ are disjoint from $\left\{z^{m-1} \in \mathbf{R}\right\}$. If $m$ is odd, there is a branch of $f^{-1}\left(h_{k}\right)$ :

$$
\left\{z \mid z=r e^{(2 k+1) \pi i /(m-1)},-\infty<r<r_{0}<-(1 / m)^{1 /(m-1)}\right\},
$$

which is contained in $l_{k}$. The other $m-1$ branches of $f^{-1}\left(h_{k}\right)$ are disjoint from $\left\{z^{m-1} \in \mathbf{R}\right\}$.

Proof. We first prove that $f^{-1}\left(h_{k}\right) \cap\left\{z^{m-1} \in \mathbf{R}\right\} \subset l_{k}$. In fact, if $z=$ $r e^{i \theta} \in f^{-1}\left(h_{k}\right) \cap\left\{z^{m-1} \in \mathbf{R}\right\}$, we have $e^{i(m-1) \theta}= \pm 1$ and there is $\rho>\rho_{0}$ such that $z^{m}+z=\rho e^{(2 k+1) \pi i /(m-1)}$, that is,

$$
r e^{i \theta}\left(1 \pm r^{m-1}\right)=\rho e^{(2 k+1) \pi i /(m-1)} .
$$

Now $\rho \neq 0$ implies $r \neq 0$ and $\left(1 \pm r^{m-1}\right) \neq 0$. Thus, the above equality shows that $z=r e^{i \theta}$ and $f(z)=\rho e^{(2 k+1) \pi i /(m-1)}$ lie on the same straight line $l_{k}$.

However,if $z=r e^{(2 k+1) \pi i /(m-1)} \in l_{k}$ with $r$ real and $z \in f^{-1}\left(h_{k}\right)$, then we have

$$
r-r^{m}=\rho \quad \text { where } \rho>\rho_{0}
$$

or

$$
\varphi_{\rho}(r)=r^{m}-r+\rho=0 .
$$

It is easy to check that when $m$ is even and $\rho>\rho_{0}$, the equation has no real root, so $f^{-1}\left(h_{k}\right) \cap\left\{z^{m-1} \in \mathbf{R}\right\}=\varnothing$.

If $m$ is odd, there is a unique real root $r_{\rho}$ of equation $\varphi_{\rho}(r)=0$ and $r_{\rho}$ belongs to the interval $\left(-\infty, r_{1}\right)$, where $r_{1}=-(1 / m)^{1 /(m-1)}$. We now want to prove that the real root $r_{\rho}$ is a one-to-one continuous function of $\rho$ when $\rho>\rho_{0}$. Suppose $\rho_{0}<\rho, \rho^{\prime}$. Then $r_{\rho}-r_{\rho}^{m}=\rho$ and $r_{\rho^{\prime}}-r_{\rho^{\prime}}^{m}=\rho^{\prime}$. We have

$$
r_{\rho^{\prime}}-r_{\rho}-\left(r_{\rho^{\prime}}^{m}-r_{\rho}^{m}\right)=\rho^{\prime}-\rho,
$$

or

$$
\left(r_{\rho^{\prime}}-r_{\rho}\right)\left(1-\sum_{k=0}^{m-1} r_{\rho^{\prime}}^{k} r_{\rho}^{m-1-k}\right)=\rho^{\prime}-\rho .
$$

Since $r_{\rho}, r_{\rho^{\prime}}$ are both less than $r_{1}=-(1 / m)^{1 /(m-1)}$, we have that $r_{\rho^{\prime}}^{k} r_{\rho}^{m-1-k}$ is more than $1 / m$ for $k=0,1, \ldots, m-1$. This means that $\sum_{k=0}^{m-1} r_{\rho^{\prime}}^{k} r_{\rho}^{m-1-k}>1$ or $1-\sum_{k=0}^{m-1} r_{\rho^{\prime}}^{k} r_{\rho}^{m-1-k}<0$. Hence $\rho^{\prime}>\rho$ implies $r_{\rho^{\prime}}<r_{\rho}$. We have thus shown that $r_{\rho}$ is a strictly monotone function for $\rho>\rho_{0}$. If we fix $\rho>\rho_{0}$ and let $\rho^{\prime}$ be sufficiently close to $\rho$, we can be sure that $r_{\rho}$ and $r_{\rho^{\prime}}$ are all less than a constant $c<r_{1}$. Then $\sum_{k=0}^{m-1} r_{\rho^{\prime}}^{k} r_{\rho}^{m-1-k}-1$ will be greater than a positive constant $\delta$ (dependent only on $\rho$ ). Hence, from the equality

$$
\left|r_{\rho^{\prime}}-r_{\rho}\right|=\frac{\left|\rho^{\prime}-\rho\right|}{\left|1-\sum_{k=0}^{m-1} r_{\rho^{\prime}}^{k} r_{\rho}^{m-1-k}\right|}
$$


it follows that $r_{\rho}$ is continuous for $\rho>\rho_{0}$. We now know that the ray line $\left\{r_{\rho} e^{(2 k+1) \pi i /(m-1)} \mid \rho>\rho_{0}\right\}$ is a branch of $f^{-1}\left(h_{k}\right)$ contained in $\{z \mid z=$ $\left.r e^{(2 k+1) \pi i /(m-1)},-\infty<r<r_{1}\right\}$. By the monotonicity and continuity of $r_{\rho}$, that branch is

$$
\left\{z \mid z=r e^{(2 k+1) \pi i /(m-1)},-\infty<r<r_{0}<r_{1}\right\},
$$

with endpoint $r_{0}$, the negative root of the equation $r-r^{m}=\rho_{0}$. And the other branches of $f^{-1}\left(h_{k}\right)$ are disjoint from $\left\{z^{m-1} \in \mathbf{R}\right\}$.

LemMA 4. The figure of $f^{-1}\left(h_{k}\right)$ is symmetrical about the straight line $l_{k}$ :

Proof. Let $z_{1}=r e^{(((2 k+1) \pi /(m-1))+\theta) i} \in f^{-1}\left(h_{k}\right)$. We will prove that $z_{2}=$ $r e^{(((2 k+1) \pi /(m-1))-\theta) i} \in f^{-1}\left(h_{k}\right)$, where $r, \theta$ are real. In fact, there is $\rho>\rho_{0}$ such that

$$
z_{1}^{m}+z_{1}=r e^{(((2 k+1) \pi /(m-1))+\theta) i}\left(1+r^{m-1} e^{(m-1) \theta i}\right)=\rho e^{(2 k+1) \pi i /(m-1)} .
$$

That is

$$
\left(r \cos \theta+r^{m} \cos m \theta\right)+i\left(r \sin \theta+r^{m} \sin m \theta\right)=\rho,
$$

or

$$
r \sin \theta+r^{m} \sin m \theta=0 .
$$

Hence

$$
\begin{aligned}
z_{2}^{m}+z_{2} & =r e^{(((2 k+1) \pi /(m-1))-\theta) i}\left(1+r^{m} e^{-(m-1) \theta i}\right) \\
& =\left(\left(r \cos \theta+r^{m} \cos m \theta\right)-i\left(r \sin \theta+r^{m} \sin m \theta\right)\right) e^{(2 k+1) \pi i /(m-1)} \\
& =\rho e^{(2 k+1) \pi i /(m-1)}=z_{1}^{m}+z_{1} .
\end{aligned}
$$

This shows the symmetry of the figure of $f^{-1}\left(h_{k}\right)$.

\section{Theorem and its proof}

Let $f(z)=z+z^{m}, m \geq 2$. The critical points (singularities) of $f^{-1}(z)$ are

$$
c_{k}=\rho_{0} e^{(2 k+1) \pi i /(m-1)}, \quad k=1,2, \ldots, m-1,
$$

where $\rho_{0}=((m-1) / m)(1 / m)^{1 /(m-1)}$, and $\infty$. Let $L=\left\{z \mid z=\rho e^{(2 k+1) \pi i /(m-1)}\right.$, $\left.\rho_{0}<\rho<+\infty, k=1,2, \ldots, m-1\right\}$. Then we can choose a single-value analytic branch of $f^{-1}$ on the domain $\mathbb{C} \backslash \bar{L}$. We have

TheOREM. Let $f(z)=z+z^{m}, m \geq 2$. Then there exists an analytic determination of $f^{-1}(z)$ in $\mathbb{C} \backslash \bar{L}$ which satisfies $f^{-1}(0)=0$, is continuous to 
$\bar{L}$ one sidedly, and is such that for every $z_{0} \in \mathbb{C}$ the sequence $z_{n}=f^{-1}\left(z_{n-1}\right)$ tends to zero as $n \rightarrow \infty$.

Proof. We choose $f^{-1}(z)$ in $\mathbb{C} \backslash \bar{L}$ that is an inverse analytic branch of $f$ satisfying $f^{-1}(0)=0$, and choose $f^{-1}$ on $\bar{L}=\bigcup_{k=1}^{m-1} h_{k}$ that maps $h_{k}$ onto one of the inverse branches of $h_{k}$ ending at $z_{k}=(1 / m)^{1 /(m-1)} e^{(2 k+1) \pi i /(m-1)}$ and $f^{-1}\left(c_{k}\right)=z_{k}$ for $k=1,2, \ldots, m-1$. Thus $f^{-1}(z)$ is well defined on C. We have $f^{-1}(z)$ is continuous when $z$ tends to $\bar{L}$ from one side of $h_{k}$. In fact, there two inverse branches of $\bar{h}_{k}$ ending at $z_{k}$. By Lemma 3 , they do not lie on $l_{k}$ and are disjoint from $\left\{z^{m-1} \in \mathbf{R}\right\}$. By Lemma 4, they are symmetrical about $l_{k}$. so they, when $z_{k}$ is added, form a curve through the point $z_{k}$ which is symmetrical about $l_{k}$ and separates $\mathbb{C}$ into two regions. For $k=1,2, \ldots, m-1$, there are $m-1$ such curves separating $\mathbb{C}$ into $m$ regions, only one of them containing the origin. Then the region containing the origin is the image domain of $\mathbb{C} \backslash \bar{L}$ under $f^{-1}$ as $f^{-1}(0)=0$. Also $f^{-1}(z)$ constructed as above is continuous to $\bar{L}$ one sidedly. Moreover, $f^{-1}(L) \cap\left\{z^{m-1} \in \mathbf{R}\right\}=\varnothing$.

Obviously, $f^{-1}(z)$ is analytic at $z=0$ with an expansion

$$
f^{-1}(z)=z-z^{m}+\cdots .
$$

Let $G=\mathbb{C} \backslash\left\{z^{m-1} \in \mathbf{R}\right\}$, which is such that $G \subset \mathbb{C} \backslash \bar{L}$. By Lemma 2, $f^{-1}(G) \subset G \subset \mathbb{C} \backslash \bar{L}$. Now $G$ is the union of $2(m-1)$ components $G_{j}, j=$ $1,2, \ldots, 2(m-1)$, each $G_{j}$ being a simply connected unbounded sector. Given $G_{j}$ for some $j, f^{-n}(z)$ is analytic in $G_{j}$ for all $n>0$. Since $f^{n}(z)$ tends to infinity uniformly for $z$ sufficiently large, there exists a region in $G_{j}$ containing no limit points of the forward orbit of any $z \in \mathbb{C}$. By Result 6 of Fatou and Julia, $\left\{f^{-n}\right\}$ is normal in $G_{j}$ and every convergent subsequence tends to a constant. By Lemma 1 , with $0<\theta<\pi / 2$ and sufficiently small $\rho>0$, $f^{-n}(z)$ tends to 0 locally uniformly in the domain $D(\theta, \rho)=\bigcup_{j=1}^{m-1} D(j, \theta, \rho)$ where

$$
\begin{aligned}
D(j, \theta, \rho)=\left\{z|0<| z \mid<\rho,-\frac{(2 j-2) \pi}{m-1}-\right. & \frac{\pi-\theta}{m-1} \\
& \left.<\arg z<-\frac{(2 j-2) \pi}{m-1}+\frac{\pi-\theta}{m-1}\right\} .
\end{aligned}
$$

Since the intersection between $G_{j}$ and $D(\theta, \rho)$ is nonempty, every convergent subsequence of $\left\{f^{-n}(z)\right\}$ tends to zero in $D(\theta, \rho) \cap G_{j}$ and so tends to zero in $G_{j}$ for $j=1,2, \ldots, 2(m-1)$. This shows that $\left\{f^{-n}(z)\right\}$ tends to zero in $G=\bigcup_{j=1}^{2(m-1)} G_{j}$.

Next, we consider the convergence of $f^{-n}(z)$ in the set $\left\{z^{m-1} \in \mathbf{R}\right\}$. If $z \in L$, then $f^{-1}(z) \in G$ from Lemmas 3,4 and the construction of $f^{-1}$. 
The above discussion shows that $f^{-n}(z)$ tends to zero as $n \rightarrow \infty$. If $z=0$ then $f^{-n}(0) \equiv 0$ for all $n>0$. We will prove that $\left\{z^{m-1} \in \mathbf{R}\right\} \backslash(L \cup\{0\})=$ $\left\{z^{m-1}>0\right\} \cup\left\{z^{m-1}<0\right\} \backslash L$ lies in the Fatou set of $f$.

By Lemma 2, $\left\{z^{m-1}>0\right\} \subset A(\infty) \subset F$. Let $R=\left\{z^{m-1}<0\right\} \backslash L=\{z \mid z=$ $\left.r e^{(2 k+1) \pi i /(m-1)}, 0<r \leq \rho_{0}, k=1,2, \ldots, m-1\right\}$. For $z=r e^{(2 k+1) \pi i /(m-1)} \in \bar{R}$, $f^{\prime}(z)=1+m z^{m-1}=1-m r^{m-1}$ so $\left|f^{\prime}(z)\right|<1$ as $0<r \leq \rho_{0}$. This implies that $\left|f^{\prime}(z)\right|<1$ as $z \in \bar{R}$ except for $z=0$. By [1, Lemma 2 and Theorem 1], we get $R \subset F, \bar{R} \cap J=\{0\}$ and $R$ contains no limit points of forward orbits of points in C. Also $\left\{z^{m-1}>0\right\} \subset A(\infty)$ contains no limit points of forward orbits. From Result 5 above the accumulation points of $\left\{f^{-n}(z)\right\}$ belong to the Julia set, for every $z \in\left\{z^{m-1} \in \mathbf{R}\right\} \backslash(L \cup\{0\})$. If $f^{-n}(z) \in\left\{z^{m-1} \in \mathbf{R}\right\} \backslash L$ for all

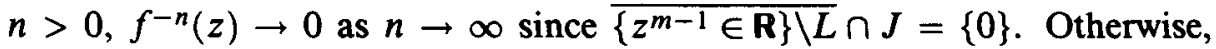
there exists an integer $n>0$ such that $w=f^{-n}(z) \notin\left\{z^{m-1} \in \mathbf{R}\right\} \backslash L$. But we have shown, for $w \notin\left\{z^{m-1} \in \mathbf{R}\right\} \backslash L$, that is, for $w \in G$ or $w \in L$, that $f^{-n}(w)$ tends to zero as $n \rightarrow \infty$. Hence $f^{-n}(z)$ also tends to zero as $n \rightarrow \infty$.

CoRollary. Let $f(z)=z+z^{m}, m \geq 2$. Then for every $z_{0} \in\left\{z|| f^{\prime}(z) \mid \leq\right.$ $1\}$, there exists a sequence $\left\{z_{n}\right\}$ such that $z_{n+1}=f\left(z_{n}\right)$ and $z_{n} \rightarrow 0, z_{-n} \rightarrow 0$ as $n \rightarrow \infty$.

Proof. This is a direct consequence of the above theorem and [1, Theorem 1].

\section{Counterexamples}

In this section we will give two examples to show that Conjecture 2 is false.

EXAMPLE 1. Let $f(z)=z(1+a z)^{2}, a>1$, be a polynomial with positive real coefficients. Now $f(-1 / a)=0 \in J$ (since $f(0)=0$ and $f^{\prime}(0)=1$ is a root of unity, from Result 3 ) and $-1 / a \in J$ (since the Julia set is completely invariant, from Result 1 ). It is easy to see that $f^{\prime}(-1 / a)=0$, so that $-1 / a$ is in $D$, one of the components of $\left\{z|| f^{\prime}(z) \mid<1\right\}$. But $J$ is a perfect set and the repulsive periodic points of $f$ are dense in $J$ from Results 1 and 2. There exists at least one repulsive periodic point $p \in D$ with period not less than 2 . Thus $f^{n}(p)$ does not converge.

Since $-1 / 2 \leq f^{\prime}(z)<1$ when $z \in[-1 / a, 0)$, we have $D \supset[-1 / a, 0)$. So the origin is a boundary point of $D$. If we restrict the initial point to be in the component of $\left\{z|| f^{\prime}(z) \mid<1\right\}$ with boundary point 0 , the result is also not true.

In this example, we showed that for a polynomial with positive real coefficients $f(z)$, the set $\left\{z|| f^{\prime}(z) \mid<1\right\}$ may contains some points in $J$. The 
next example shows that there exists such a polynomial for which there is a region in $\left\{z|| f^{\prime}(z) \mid<1\right\} \cap F$ in which iterative sequences of all points are divergent.

Let $z_{0} \in C$ be a fixed point of polynomial $f(z)$, and suppose that $\lambda=$ $f^{\prime}\left(z_{0}\right)=e^{2 \pi i \omega}$. Then we have

LemMa 5 (Siegel [7]). Let $\omega$ be an irrational. Suppose there are positive constants $a$ and $b$ satisfying $|\omega-(m / n)|>a / n^{b}$ for all integers $m, n$ with $n \geq 1$. Then there exists a neighbourhood $U$ of $z_{0}$ and a homeomorphism $\varphi: U \rightarrow D_{r}=\{\zeta|| \zeta \mid<r\}, \varphi\left(z_{0}\right)=0$, such that $\varphi \circ f \circ \varphi^{-1}(\zeta)=e^{2 \pi i \omega} \zeta$.

The set of $\omega$ satisfying the condition of Lemma 5 is dense in interval $[0,1]$.

We will construct a polynomial $f(z)$ satisfying the condition of Conjecture 2 , which has a fixed point $z_{0}$ different from 0 and is such that $\lambda=f^{\prime}\left(z_{0}\right)=$ $e^{2 \pi i \omega}$, where $\omega$ satisfies the condition of Lemma 5. For $g(\zeta)=e^{2 \pi i \omega} \zeta: D_{r} \rightarrow$ $D_{r}$, when $\zeta_{1} \in D_{r}$ and $\zeta_{1} \neq 0$, its iterative sequence $\left\{\zeta_{n}\right\}, \zeta_{n}=g\left(\zeta_{n-1}\right)=$ $e^{2 n \pi i \omega} \zeta_{1}$ is dense on circle $\left\{|\zeta|=\left|\zeta_{1}\right|\right\}$. Thus $\zeta_{n}$ does not converge as $n \rightarrow \infty$, and therefore, for $z_{1}=\varphi^{-1}\left(\zeta_{1}\right), z_{1} \neq z_{0}, z_{n+1}=f\left(z_{n}\right)$ is also not convergent as $n \rightarrow \infty$. Since $\left|f^{\prime}\left(z_{0}\right)\right|=1$, we deduce, using the minimum principle, that there is a region $V$ in $U$ disjoint from $z_{0}$ such that for all $z \in V,\left|f^{\prime}(z)\right|<1$. This is all we need.

EXAMPLE 2. Choose $\omega \in[0,1]$, satisfying the condition of Lemma 5 . Let $\theta=(2+\omega) / 4$. Then $\pi<2 \pi \theta<3 \pi / 2$ or $\cos 2 \pi \theta<0$. Let $r=$ $\left(\left|e^{2 \pi i \omega}-1\right| /\left|e^{4 \pi i \theta}-1\right|\right)^{1 / 3}$. Let

$$
\begin{aligned}
f(z) & =z+z^{2}\left(z-r e^{2 \pi i \theta}\right)\left(z-r e^{-2 \pi i \theta}\right) \\
& =z+r^{2} z^{2}-2 r \cos (2 \pi \theta) z^{3}+z^{4} .
\end{aligned}
$$

Then $f(z)$ is a polynomial with positive real coefficients having nonzero fixed point $z_{0}=r e^{2 \pi i \theta}$.

$$
\begin{aligned}
f^{\prime}\left(z_{0}\right) & =1+2 r^{2} z_{0}-3 r\left(e^{2 \pi i \theta}+e^{-2 \pi i \theta}\right) z_{0}^{2}+4 z_{0}^{3} \\
& =1+r^{3} e^{2 \pi i \theta}\left(e^{4 \pi i \theta}-1\right) .
\end{aligned}
$$

Since $e^{i \alpha}-1=\left|e^{i \alpha}-1\right| e^{(\pi+\alpha) i / 2}$ for real $\alpha$,

$$
r^{3} e^{2 \pi i \theta}\left(e^{4 \pi i \theta}-1\right)=r^{3} e^{2 \pi i \theta}\left|e^{4 \pi i \theta}-1\right| e^{(4 \pi \theta+\pi) i / 2} .
$$

when $\theta=(2+\omega) / 4$ and $r=\left(\left|e^{2 \pi i \omega}-1\right| /\left|e^{4 \pi i \theta}-1\right|\right)^{1 / 3}$, we get

$$
\begin{aligned}
f^{\prime}\left(z_{0}\right) & =1+\left|e^{2 \pi i \omega}-1\right| e^{(8 \pi((2+\omega) 14)+\pi) i / 2} \\
& =1+\left|e^{2 \pi i \omega}-1\right| e^{(2 \pi \omega+\pi) i / 2}=e^{2 \pi i \omega} .
\end{aligned}
$$


This completes the construction of our example.

\section{Acknowledgements}

I should like to thank Fuyao Ren for considerable discussion, and the referee for his suggestions and help.

\section{References}

[1] Zalman Rubinstein, 'A variational method for the construction of convergent iterative sequences', J. Austral. Math. Soc. Ser. A 41 (1986), 51-58.

[2] H. Brolin, 'Invariant sets under iteration of rational functions', Ark. Mat. 6 (1965), 103144.

[3] P. Fatou, 'Sur les équations fonctionelles', Bull. Soc. Math. France 47 (1919), 161-271.

[4] P. Fatou, 'Sur l'itération des fonctions transcendantes entieres', Acta Math. 47 (1926), 337-370.

[5] G. Julia, 'Memoire sur l'itération des fonctions rationnelles', J. Math. Pures Appl. (8) 1 (1918), 47-245.

[6] I. N. Baker, 'Permutable power series and regular iteration', J. Austral. Math. Soc. 2 (1962), 265-194.

[7] C. Seigel, 'Iteration of analtyic functions', Ann. of Math. (2) 43 (1942), 607-612.

Institute of Mathematics

Fudan University

Shangai

People's Republic of China 